\title{
Perfil dos pacientes atendidos em urgência e emergência cardiológica em um serviço de referência
}

\author{
Eduardo Tavares Gomes*, Andrey Vieira de Queiroga**, Simone Maria Muniz da Silva Bezerra, D.Sc.***
}

\begin{abstract}
*Enfermeiro, especialista em Suporte Avançado de Vida pela Faculdade de Enfermagem Nossa Senhora das Graças da Universidade de Pernambuco, residente de Enfermagem em Cardiologia do Pronto-Socorro Cardiológico Universitário de Pernambuco, **Enfermeiro, especialista em Obstetrícia pela Faculdade Santa Emília - FASER, residente de Enfermagem em Cardiologia do Pronto-Socorro Cardiológico Universitário de Pernambuco, ${ }^{* * *}$ Enfermeira, pós-doutora em Enfermagem Fundamental pela Escola de Enfermagem de Ribeirão Preto da Universidade de São Paulo, professora orientadora do Programa Associado de Pós-graduação em Enfermagem UPE/UEPB, coordenadora da especialização em Enfermagem em Cardiologia na modalidade residência do Pronto-Socorro Cardiológico de Pernambuco
\end{abstract}

\begin{abstract}
Resumo
Objetivo: Verificar o perfil dos pacientes admitidos em um serviço de emergência de referência de atenção à saúde. Material e métodos: Trata-se de um estudo de corte transversal, prospectivo, de abordagem quantitativa realizado em um serviço de emergência de referência de Recife/PE. Participaram da amostra 91 pacientes admitidos no serviço de emergência, no período após classificação de risco e o período da internação hospitalar ou alta, antes de completadas 24 horas da entrada no serviço. Resultados: A amostra apresentou predominância do sexo masculino $(53,8 \%)$, com idade maior que 60 anos $(60,4 \%)$, procedente da própria capital $(41,8 \%)$, aposentados $(46,2 \%)$, mais de 10 anos de escolaridade $(57,2 \%)$, renda entre 3 e 6 salários mínimos (35,6\%). Predominância de hipertensão de grau I e queixa principal a dor torácica $(41,8 \%)$. A média de tempo entre início dos sintomas e admissão foi de 18,71 $\pm 13,09$. A maior parte dos pacientes chegou ao serviço entre 12 e 24 horas (31,9\%), através de ambulâncias de serviços de atendimento pré-hospitalares fixos (75,8\%), 13,2\% foram removidos pelo SAMU e 11\% foram transferidos ao hospital por transporte próprio. Conclusão: Os profissionais dos serviços de emergência devem estar preparados para prestar assistência ideal, visando melhora do prognóstico e aumentar a sobrevida dos pacientes.
\end{abstract}

Palavras-chave: perfil de saúde, serviços médicos de emergência, doenças cardiovasculares, cardiologia.

\section{Abstract \\ Profile of patients attending urgency and emergency cardiology in a reference service}

Objective: To determine the profile of patients admitted to a health care emergency referral service. Methods: This is a cross-sectional study, prospective cohort, and quantitative approach carried out in an emergency referral service of

Recebido em 23 de dezembro de 2013; aceito em 16 de abril de 2014. Endereço para correspondência: Eduardo Tavares Gomes, Pronto-Socorro Cardiológico Universitário de Pernambuco, Universidade de Pernambuco, Rua dos Palmares, 262, Santo Amaro, 50100-060 Recife PE, E-mail: andreyquixa@hotmail.com,simonemunizm2@gmail.com 
Recife/PE. A sample of 91 patients admitted to the emergency department, in the period following risk classification and during hospital admission or discharge, before completing 24 hours of admission in the service. Results: The sample was predominantly male $(53.8 \%)$, over 60 years $(60.4 \%)$, coming from Recife $(41.8 \%)$, retired $(46.2 \%)$, more than 10 years of schooling (57.2\%), income between 3 and 6 minimum wages (35.6\%). Prevalence of hypertension grade I and main chest pain complaint $(41.8 \%)$. The average time between onset of symptoms and admission was $18.71 \pm 13.09$. The majority of patients arrived at health care emergency service between 12 and 24 hours (31.9\%), by pre-hospital ambulance care services (75.8 \%), 13.2\% were removed by SAMU (Mobile Emergency Care Service) and 11\% were transferred to hospital using their own transport. Conclusion: The emergency care services personnel should be able to provide optimal care, aiming improvement of prognosis and increase survival of patients.

Key-words: health profile, emergency medical service, cardiovascular diseases, cardiology.

\section{Resumen}

\section{Perfil de pacientes atendidos en urgencia y emergencia cardiológica en un servicio de referencia}

Objetivo: Verificar el perfil de pacientes ingresados en un servicio de referencia de emergencia para la atención a la salud. Métodos: Se trata de un estudio transversal, prospectivo de cohortes, y enfoque cuantitativo, realizado en un servicio de referencia de emergencia de Recife -PE. Participaron del estudio 91 pacientes ingresados en el servicio de urgencias, en el período que siguió a la clasificación de riesgo y durante el ingreso y alta hospitalaria, antes de completar las 24 horas. Resultados: La muestra fue predominantemente masculina $(53,8 \%)$, con más de 60 años $(60,4 \%)$, procedentes de Recife (41,8\%), jubilados (46,2\%), más de 10 años de estudio (57,2\%), ingresos entre 3 y 6 salarios mínimos (35,6\%). Predominio de hipertensión de grado I y principal queja dolor en el pecho $(41,8 \%)$. El tiempo promedio entre el inicio de síntomas y el ingreso fue de 18,71 $\pm 13,09$. La mayoría de los pacientes se presentaron para el tratamiento entre $12 \mathrm{y}$ 24 horas $(31,9 \%)$ por ambulancia de los servicios de atención prehospitalaria $(75,8 \%)$, el 13,2 \% fueron recogidos por el SAMU (Sistema de Atención Móvil de Urgencias) y el 11\% fue trasladado al hospital por sus medios propios. Conclusión: Los servicios de emergencia profesionales deben ser capaces de proporcionar una atención óptima a los pacientes, con el objetivo de mejora del pronóstico y aumentar la supervivencia de los pacientes.

Palabras-clave: perfil de salud, servicios médicos de urgencias, enfermedades cardiovasculares, cardiología.

\section{Introdução}

A Política Nacional de Atenção às Urgências orienta a organização do sistema de saúde em unidades pré-hospitalares fixas e móveis, unidades hospitalares e pós-hospitalares. Essas instâncias devem estar preparadas para suas atribuiçóes no atendimento ao paciente agudo e integradas entre si através de uma unidade de regulação que organize o atendimento dos pacientes dentro de suas necessidades, em tempo hábil, distribuindo-os na rede até o atendimento especializado, quando necessário [1].

Diante do preconizado, várias regióes metropolitanas vêm passando pela reorganização da rede, com o fortalecimento das unidades pré-hospitalares móveis, representada pelo Serviço de Atendimento Móvel de Urgência (SAMU); implantação e preparo de serviços de atendimento pré-hospitalar fixo, em especial as Unidades não-hospitalares de Atendimento às Urgências ou Unidades de Pronto Atendimento e mudança no atendimento ao público das unidades hospitalares. Os hospitais especializados, há anos referência para a população em atendimento de emergência, passaram a receber pacientes encaminhados pela regulação médica e não mais atender ao público irrestritamente, direcionando o primeiro atendimento e caracterizando as unidades pré-hospitalares como porta de entrada na rede. Porém, a Política Nacional de Atenção às Urgências (PNAU) ainda é incipiente, pois não permite, todavia, a observação de sua efetividade [2].

No que tange ao atendimento às urgências cardiovasculares e cerebrais, por se tratarem de acometimentos com resolução tempo-dependente, a necessidade de organizaçáo torna-se mais premente. No entanto, apesar do suporte especializado ser decisivo e indispensável na fase aguda do infarto agudo do miocárdio, síndromes coronarianas, acidente vascular cerebral e entidades clínicas, o atendimento inicial precoce e a primeira abordagem realizada são decisivos em relação ao desfecho e ao prognóstico. Por ser reconhecido, entâo, que as unidades pré-hospitalares 
devem estar preparadas para esse atendimento primário, visto que são as portas de entrada no sistema, o Ministério da Saúde estabeleceu a atenção às urgências cardiovasculares e cerebrovasculares como linhas de cuidado prioritárias [3].

Para a garantia do tempo terapêutico do atendimento ao infarto agudo do miocárdio e às síndromes coronarianas agudas, a Linha do Cuidado do Ministério da Saúde reforça, como prioridade, as açóes: implantação de protocolos de transferência e transporte para agilizar o atendimento - início rápido do tratamento de reperfusáo imediata dos pacientes; expansão da telemedicina para diagnóstico eletrocardiográfico precoce (expansão do Tele ECG nos SAMU e nas UPA); qualificação do atendimento ao infarto nas urgências pré-hospitalares (SAMU e UPA); implementação da integração entre o diagnóstico pré-hospitalar e a conduta hospitalar; melhoria da comunicação e a articulação entre a Central de Regulação Médica de Urgência e as unidades de terapia intensiva coronariana para o atendimento imediato; além da garantia do fornecimento de medicamentos essenciais ao tratamento do IAM [3].

Através desta pesquisa, objetivou-se verificar o perfil dos pacientes admitidos em um serviço de emergência de referência que integra a rede de atenção à saúde de uma capital.

\section{Material e métodos}

Trata-se de um estudo de corte transversal, descritivo-exploratório, prospectivo, de abordagem quantitativa. Foi realizado no serviço de emergência de referência na cidade Recife-PE, que mantém atendimento cardiológico $24 \mathrm{~h}$. A pesquisa se deu entre dezembro de 2012 e fevereiro de 2013. Participaram da amostra 91 pacientes admitidos no serviço de emergência, no período após a triagem, acolhimento e classificação de risco, realizados por enfermeiros, e o período da internaçáo hospitalar ou alta, antes de completadas 24 horas da entrada no serviço. Foram excluídos pacientes que se recusaram a participar do estudo em qualquer momento da pesquisa; pacientes inconscientes, desorientados ou confusos de forma a comprometer sua autonomia e com comunicação prejudicada que dificulta a coleta de dados, muitos deles classificados pelos enfermeiros como vermelhos, que segundo o protocolo institucional, representavam o risco mais elevado e a necessidade de atendimento em menor tempo possível.
O instrumento de coleta foi elaborado contendo dados clínicos relativos aos antecedentes de saúde e os diagnósticos de enfermagem possíveis de serem aplicados. Os dados foram coletados através de entrevista com o paciente e para outras informaçóes foram consultados o resumo da classificação de risco realizada pelo enfermeiro e a ficha de atendimento médico. Os dados coletados foram arquivados em banco de dados e submetidos à análise estatística utilizando o software Epi-info 6.04. A realização deste trabalho teve a apreciaçáo e aprovação do Comitê de Ética em Pesquisa do Instituto de Medicina Integral de Pernambuco (IMIP).

\section{Resultados}

A amostra apresentou predominância do sexo masculino (53,8\%), com idade maior que 60 anos $(60,4 \%)$, procedência principal a própria capital $(41,8 \%)$, aposentados (46,2\%), mais de 10 anos de escolaridade $(57,2 \%)$ e renda entre 3 e 6 salários mínimos $(35,6 \%)$ (Tabela I).

Tabela I - Caracterização sociodemográfica dos pacientes atendidos em uma emergência cardiológica de referência. Recife-PE, 2012.

\begin{tabular}{|c|c|c|}
\hline Variáveis & $N=91$ & $\%$ \\
\hline \multicolumn{3}{|l|}{ Sexo } \\
\hline Masculino & 49 & 53,8 \\
\hline Feminino & 42 & 46,2 \\
\hline \multicolumn{3}{|l|}{ Idade } \\
\hline$<40$ anos & 4 & 4,4 \\
\hline De 40 a 49 anos & 5 & 5,5 \\
\hline De 50 a 59 anos & 27 & 29,7 \\
\hline De 60 a 69 anos & 35 & 38,4 \\
\hline$>70$ anos & 20 & 22,0 \\
\hline \multicolumn{3}{|l|}{ Procedência } \\
\hline Interior & 19 & 20,9 \\
\hline Capital & 38 & 41,8 \\
\hline RMR & 34 & 37,4 \\
\hline \multicolumn{3}{|l|}{ Atividade laboral } \\
\hline Desempregado & 19 & 20,9 \\
\hline Aposentado & 42 & 46,2 \\
\hline De 20 a $40 h / s e m$ & 16 & 17,6 \\
\hline Mais de $40 \mathrm{~h} / \mathrm{sem}$ & 14 & 15,4 \\
\hline \multicolumn{3}{|l|}{ Escolaridade } \\
\hline Analfabeto & 5 & 5,5 \\
\hline Até 4 anos & 14 & 15,4 \\
\hline De 5 a 10 anos & 20 & 22,0 \\
\hline De 10 a 14 anos & 27 & 29,7 \\
\hline Mais de 15 anos & 25 & 27,5 \\
\hline
\end{tabular}




\begin{tabular}{lcc}
\hline Renda & 15 & 16,5 \\
\hline Até 3 salários & 32 & 35,2 \\
De 3 a 6 salários & 31 & 34,1 \\
De 6 a 10 salários & 3 & 3,3 \\
Mais de 10 salários & 10 & 11,0 \\
Não refere & \\
\hline
\end{tabular}

Quanto à apresentação clínica a triagem, houve predominância de hipertensão de grau I, índice de massa corporal compatível a peso normal $(58,2 \%)$ e queixa principal a dor torácica $(41,8 \%)$, seguida por dispneia $(20,9 \%)$ e náuseas $(17,6 \%)$ (tabela II). A referência de dor torácica como queixa incluiu a dor retroesternal típica e as irradiaçôes. Não foi considerada a classificação da dispneia. A média de tempo entre início dos sintomas e admissáo ao serviço foi de 18,71 $\pm 13,09$. Poucos pacientes chegaram à porta do serviço com tempo de início dos sintomas menores que 1 hora $(6,6 \%)$, sendo que a maior parte chegou entre 12 e 24 horas $(31,9 \%)$ e entre 24 e 48 horas $(29,7 \%)$ (tabela II). A maior parte dos pacientes chegou ao serviço através de ambulâncias de serviços de atendimento pré-hospitalares fixos $(75,8 \%), 13,2 \%$ foram removidos pelo Serviço de Atendimento Móvel de Urgência (SAMU) e apenas $11 \%$ foi transferido do local de início dos sintomas ao hospital por transporte próprio. Quanto à origem, 58,24\% foram regulados pelas unidades de saúde pré-hospitalares, enquanto que $29,67 \%$ deram entrada no serviço proveniente do próprio domicílio e 12,09 da via pública, que no estudo inclui espaços coletivos de lazer, comércio e conhecimentos (tabela II).

Tabela II - Distribuição dos pacientes atendidos em uma emergência cardiológica de referência quanto à apresentação clínica à triagem. Recife/PE, 2012.

\begin{tabular}{lcc}
\hline \multicolumn{1}{c}{ Níveis Pressóricos } & $\mathrm{N}=91$ & $\%$ \\
\hline Ótima & 1 & 1,10 \\
Normal & 9 & 9,89 \\
Limítrofe & 21 & 23,08 \\
Estágio 1 & 31 & 34,07 \\
Estágio 2 & 22 & 24,18 \\
Estágio 3 & 4 & 4,40 \\
\hline \multicolumn{1}{c}{$\mathrm{IMC}$} & & \\
\hline Peso normal $\left(18,5-24,9 \mathrm{~kg} / \mathrm{m}^{2}\right)$ & 53 & 58,2 \\
Sobrepeso $\left(25-29 \mathrm{~kg} / \mathrm{m}^{2}\right)$ & 35 & 38,5 \\
Obesidade $\left(>30 \mathrm{~kg} / \mathrm{m}^{2}\right)$ & 3 & 3,3 \\
Queixa principal & & \\
Dor torácica & 38 & 41,8 \\
Dispneia & 19 & 20,9 \\
Náusea & 16 & 17,6
\end{tabular}

\begin{tabular}{lcc} 
Vômito & 9 & 9,9 \\
Outras queixas & 5 & 5,5 \\
Síncope & 4 & 4,4 \\
\hline \multicolumn{2}{l}{ Tempo entre início dos sintomas e admissão } & \\
\hline Menos de $1 \mathrm{~h}$ & 6 & 6,6 \\
Entre 1 e 6 h & 10 & 11,0 \\
Entre 6 e 12 h & 19 & 20,9 \\
De 12 a 24 h & 29 & 31,9 \\
Entre 24 h e 48 h & 27 & 29,7 \\
\hline Meio de transporte & & \\
\hline Próprio & 10 & 11,0 \\
Ambulância (de outros serviços & & \\
pré-hospitalares fixos) & 69 & 75,8 \\
SAMU & 12 & 13,2 \\
\hline Origem & & \\
\hline Domicílio & 27 & 29,67 \\
Via pública & 11 & 12,09 \\
Outra unidade de saúde & 53 & 58,24 \\
\hline
\end{tabular}

\section{Discussão}

O sexo masculino e a faixa etária maior que 65 anos encontradas estáo relacionada ao risco de doenças cardiovasculares e ao atendimento de emergência [4]. Os pacientes que constituíram a amostra náo foram segmentados por patologia ou condição clínica, incluindo-se vários diagnósticos médicos de entidades nosológicas cardiológicas.

Os pacientes que se apresentam nas unidades de emergência com dor torácica, devem ter uma abordagem adequada [5]. As ferramentas clínicas utilizadas para diferenciar a origem da dor torácica são: a adequada e rápida anamnese, exame clínico específico e direcionado, e averiguação dos sinais vitais [6]. O quadro sintomatológico da dor torácica é clássico e geralmente é descrita como um peso ou aperto no tórax, uma "queimação" ou dificuldade de respirar. Frequentemente está associada a uma irradiação para o ombro esquerdo, pescoço, regiáo dorsal ou entre as escápulas e braço. Tipicamente ela vai aumentando em intensidade durante um período de poucos minutos [7]. A dor retroesternal pode afetar de forma significativa a qualidade de vida dos pacientes e frequentemente proporcionam modificaçóes e limitaçóes de seu estilo de vida [8]. Os pacientes que se apresentam nas unidades de emergência com dor torácica, devem ter uma abordagem adequada. A dor torácica configurou nesta pesquisa com a queixa mais frequente encontrada no serviço de emergência, enfocando a necessidade de protocolo mais efetivo e consistente para a reabilitaçáo dos pacientes. 
No estudo, a dispneia não foi avaliada por meio de nenhuma classificação, sendo apresentada de forma generalizada. Outros autores observaram a dispneia como o principal sintoma associado à insuficiência cardíaca atendida de emergência, estando relacionada às alteraçóes na circulação pulmonar e diminuição do débito cardíaco [9].

Alguns estudos têm demonstrado que o tempo médio de o início dos sintomas e a chegada ao hospital dos pacientes com infarto agudo do miocárdio é de 2 a 3 horas. $\mathrm{O}$ retardo intra-hospitalar, que é o intervalo de tempo entre a chegada do paciente e o diagnóstico médico com a consequente iniciação terapêutica específica, é outro problema que atinge a maioria dos hospitais em todo o mundo. As unidades de dor torácica podem estar localizadas dentro ou adjacente à sala de emergência, como uma verdadeira área física ou somente como um processo de ação. $\mathrm{O}$ essencial é que exista um grupo de profissionais treinados e qualificados atuando de maneira sincrônica, visando prioridades, rapidez, eficiência, alta qualidade e contençáo de custo [10].

Um estudo realizado para avaliar o tempo decorrido entre o início dos sintomas e a admissão a porta da emergência em uma unidade de Porto Alegre, realizado em 2008, ou seja, antes da proposta de reorganização do atendimento às urgências e emergências, avaliou que os pacientes com IAM apresentavam tempo médio de $3 \mathrm{~h} 59 \mathrm{~min} \pm 2 \mathrm{~h} 55 \mathrm{~min}$. Dos pacientes da amostra, $59,8 \%$ chegaram à emergência com automóvel próprio e em menor tempo [4]. Outro estudo, realizado em 2007, mostrou que cerca de dois terços dos pacientes, ao apresentarem os primeiros sinais e sintomas, procuraram diretamente atendimento a unidade hospitalar [11]. O nosso estudo mostra uma mudança no perfil do atendimento, visto que a porta de entrada passa a ser a unidade pré-hospitalar fixa e os pacientes encaminhados para o serviço de referência, chegando $75,8 \%$ pela remoção em ambulâncias de unidades fixas e $13,2 \%$ pelo SAMU.

Não foi avaliada relação estatística entre o tempo de atendimento e a localização geográfica, mas infere-se que a predominância da procedência na capital e região metropolitana diminui o tempo para acesso ao serviço.

Outros autores referem que os serviços de saúde não parecem preparados para o atendimento de urgências e emergências cardiológicas e referem a necessidade de capacitação e habilitação ao tratamento precoce, como o uso de trombolíticos nas unidades pré-hospitalares [11]. Estas unidades devem ter protocolos estabelecidos e divulgados entre o time assistencial para garantir que o atendimento se dê baseado em evidências, com eficiência e eficácia [12].

\section{Conclusão}

Em virtude dessas mudanças, é premente a necessidade de capacitação das unidades pré-hospitalares para a atenção nessas situações, visto que se trata de situaçóes tempo-dependentes e que o arranjo da rede de atenção às urgências e emergências pode aumentar o tempo do início dos sintomas até o acesso a terapêutica de reperfusão.

Além disso, enfermeiros de unidades de emergência hospitalar devem estar treinados e preparados para atender a dor torácica, guiados por protocolos baseados em evidências, considerando a necessidade de diminuir o retardo intra-hospitalar.

\section{Referências}

1. Ministério da Saúde. Política Nacional de Atenção às Urgências. Brasília (DF), 2006.

2. Luz CC, Junger WL, Cavalini LT. Análise da atenção pré-hospitalar ao acidente vascular cerebral e ao infarto agudo do miocárdio na populaçáo idosa de Minas Gerais. Rev Assoc Med Bras 2010;56(4):452-7.

3. Ministério da Saúde. Secretaria de Atenção à Saúde. Departamento de Atençáo Especializada. Manual Instrutivo da Rede de Atençáo às Urgências e Emergências no Sistema Único de Saúde (SUS). Brasília: Ministério da Saúde; 2013. 84p.

4. Franco B, Rabelo ER, Goldmeyer S, Souza EN. Pacientes com infarto agudo do miocárdio e os fatores que interferem na procura por serviço de emergência: implicaçôes para a educação em saúde. Rev Latinoam Enferm 2008;16(3).

5. Ferreira AMC, Madeira MZA. A dor torácica na sala de emergência: uma revisão da literatura. Rev Interdisc NOVAFAPI 2011;4(1):50-6.

6. Massaglia MT, Neris ES, Silva MLT. Uso do protocolo da dor torácica em pronto atendimento de hospital de referência em cardiologia. Rev Bras Cardiol 2013;26(5):374-81.

7. Ministério da Saúde. Secretaria de Atenção à Saúde. Departamento de Atenção Básica. Acolhimento à demanda espontânea: queixas mais comuns na Atenção Básica/Ministério da Saúde, Secretaria de Atenção à Saúde, Departamento de Atenção Básica. 1. ed. 1. reimp. - Brasília: Ministério da Saúde; 2013. 
8. Domingues GRS, Moraes Filho JPP. Dor torácica não-cardiogênica. Arq Gastroenterologia 2009;46(3):2339.

9. Aliti GB, Linhares JCC, Linch GFC, Ruschel KB, Rabelo ER. Sinais e sintomas de pacientes com insuficiência cardíaca descompensada: inferência dos diagnósticos de enfermagem prioritários. Rev Gaúcha Enferm 2011;32(3):590-5.

10. Ouriques BF. Unidades de dor torácica: revisão da literatura [TCC]. Rio de Janeiro: Escola de Saúde do Exercito; 2008. p.8-29.
11. Mussi FC, Passos LCS, Menezes AA, Caramelli B. Entraves no acesso à atenção médica: vivências de pessoas com infarto agudo do miocárdio. Rev Assoc Med Bras 2007;53(3):234-39.

12. Escosteguy CC, Teixeira AF, Portela MC, Guimarães AEC, Lima SML, Ferreira VMB. Implementando diretrizes clínicas na atenção ao infarto agudo do miocárdio em uma emergência pública. Arq Bras Cardiol 2011;96(1):18-25.

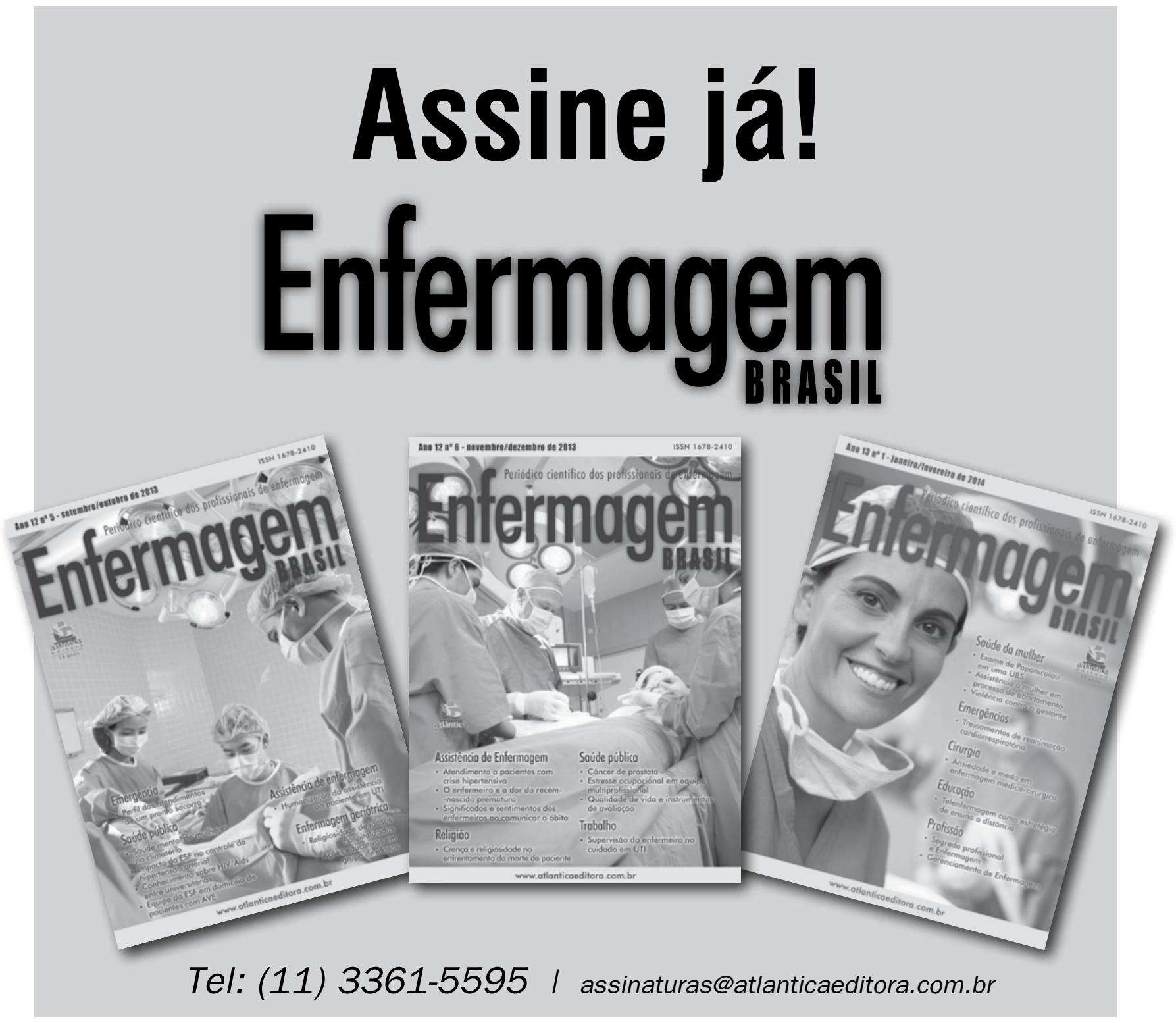

